\title{
Strong Minimum Energy Topology in Wireless Sensor Networks: NP-Completeness and Heuristics
}

\author{
Xiuzhen Cheng, Member, IEEE, Bhagirath Narahari, Rahul Simha, Member, IEEE, \\ Maggie Xiaoyan Cheng, Member, IEEE, and Dan Liu
}

\begin{abstract}
Wireless sensor networks have recently attracted lots of research effort due to its wide range of applications. These networks must operate for months or years. However, the sensors are powered by battery, which may not be possible to be recharged after they are deployed. Thus, energy-aware network management is extremely important. In this paper, we study the following problem: Given a set of sensors in the plane, assign transmit power to each sensor such that the induced topology containing only bidirectional links is strongly connected. This problem is significant in both theory and application. We prove its NP-Completeness and propose two heuristics: power assignment based on minimum spanning tree (denoted by MST) and incremental power. We also show that MST heuristic has a performance ratio of 2 . Simulation study indicates that the performance of these two heuristics does not differ very much, but, in average, the incremental power heuristic is always better than MST.
\end{abstract}

Index Terms-Minimum energy topology, power control, wireless sensor networks, NP-Completeness, incremental power heuristic.

\section{INTRODUCTION}

$\mathrm{T}$ HE research in wireless sensor networks has rapidly grown in recent years, especially when the technologies of sensor, actuator, and radio become more and more mature [3], [11], [21]. Sensor network has many applications, ranging from civil, such as smart classroom, medical monitoring, and monitoring of natural habitats and ecosystems, to military, such as security and surveillance, etc. One of the most important features of wireless sensor networks is the extreme energy constraint [19], [21]. In this paper, we are going to consider the following problem: What is the lower bound of the total energy consumed by all sensors when they form a networked collaboration system? This problem is significant in both theory and application.

A sensor network provides a global view of the monitored object area based on many local observations. The local information must be disseminated for further processing to complete the job. Thus, global connectivity should be maintained for proper data propagation. In a sensor network or any other all-wireless network, the connection between any two devices is controlled by their transmit powers. If sensor $A$ can correctly decode the signal from sensor $B$, then there exists a unidirectional link from $B$ to $A$; otherwise, $A$ is unreachable from $B$. If unidirectional links from both $A$ to $B$ and $B$ to $A$ exist, then the link between $A$ and $B$ is bidirectional. Usually, a sensor network

- X. Cheng, B. Narahari, and R. Simha are with the Department of Computer Science, The George Washington University, Washington, DC 20052. E-mail: \{cheng, narahari, simha\}@gwu.edu\}.

- M. Xiaoyan Cheng and D. Liu are with the Department of Computer Science and Engineering, University of Minnesota, Minneapolis, MN 55455. E-mail: \{xiaoyan, danliu\}@cs.umn.edu\}.

Manuscript received 23 Nov. 2002; revised 16 Apr. 2003; accepted 2 June 2003.

For information on obtaining reprints of this article, please send e-mail to: tmc@computer.org, and reference IEEECS Log Number 11-112002. contains both unidirectional and bidirectional links. And, these links must form a globally connected network. Minimizing the total transmit power to maintain global connectivity is a network optimization problem that attracts a lot of attention [6], [9], [17].

There exist several variations of the problem formulation, based on whether unidirectional links are allowed or not, and how strong we want the connectivity to be. For example, we may require that all bidirectional links form a strongly connected graph (a graph with at least one path from any node to any other node) or we relax this requirement to allow unidirectional links. We may want to compute a $k$-connected graph (a graph with at least $k$ disjoint paths from any node to any other node), where $k>1$ for network survivability; or, we simply ask for $k=1$. We may even upper bound the degree of each node (the number of one-hop neighbors) to balance the network load. The problem we study in this paper considers only bidirectional links and the resultant topology is strongly connected. The formal definition of the problem is stated below:

Definition 1 (Strong Minimum Energy Topology (SMET) Problem). Given a set of sensors in the plane, compute the transmit power of each sensor, such that there exists at least one bidirectional path (containing only bidirectional links) between any pair of sensors and the sum of all the transmit powers is minimized.

\section{Remarks.}

1. Note that, in this paper, "sensor" and "node" are exchangeable. By convention, in the context of sensor networks, we use "sensor," while in the context of graph topology, we use "node." 
2. We assume all sensors are located in the twodimensional plane.

3. The power function or the transmit power for sensor $u$ to reach sensor $v$ is denoted by $t \cdot d^{\alpha}$, where $d$ is the distance between $u$ and $v, t$ is the threshold which is a function of the signal-tonoise ratio at $v$, and $\alpha$ is a constant that is related to path loss. In a typical application environment, $\alpha$ is from two to four [26].

4. For better exposition, we use $f(d)$ to refer to the power function assigned to a node with transmission distance $d$.

Note that our problem formulation is different than those in [6], [9], [17], which focus on strong connectivity with unidirectional links. We denote their problem by gSMET. In other words, gSMET seeks a power assignment for an ad hoc network such that the total power is minimized and the topology is strongly connected. The resultant topology in gSMET may contain unidirectional links. The optimal solution of SMET is no less than that of gSMET, for the same given network. This is because only bidirectional link will be considered in SMET. Chen and Huang [6] and Clementi et al. [9] have proven that gSMET is NP-Complete. Both references show that the minimum cost spanning tree (with edge length as the cost) has a performance ratio of 2 . While gSMET and SMET are two variations of the general minimum energy topology problem mentioned above, their NP-Completeness proofs are totally different. The reduction schemes used in [6] and [9] are not applicable to SMET. We are going to use the "unidirectional graph model" [17] in our NP-Completeness proof as we only consider bidirectional links, while [6] and [9] use the "directed graph model" [17], as they consider both unidirectional and directional links.

Our problem formulation is also based on the following observations:

1. Even though unidirectional links exist in sensor networks, current MAC layer protocols such as IEEE 802.11 [15] and S-MAC [31] only take bidirectional links into consideration. And, it seems that a MAC layer protocol, which efficiently supports asymmetric links, may not exist in the near future [22]. The promising network routing protocols for multihop system such as DSR [14] and AODV [20] assume 802.11 as their MAC layer protocol. AODV itself even only works with bidirectional links.

2. A higher transmit power incurs higher interference to the surrounding devices, thus decreasing network throughput [26]. A transmit power strong enough for the receiver to correctly decode data signal is ideal in multihop wireless networks.

3. The solution to our problem and other variations of the problem can provide reference to sensor network deployment. For example, the heuristics proposed in this work can serve as a theoretical guide for determining strongly related key parameters such as sensor density and transmit power [28].

4. Our work also provides reference to scientists who depend on network simulators for their research. For example, with either GloMoSim [2] or NS2 [1], the two most popular wireless network simulators,

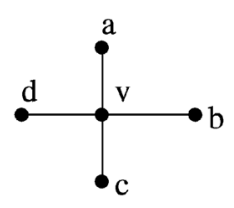

(a)

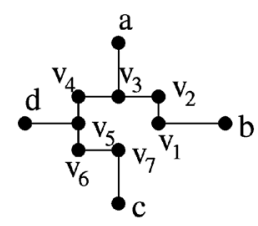

(b)
Fig. 1. Each degree-4 vertex (vertex $v$ in (a)) is replaced with a widget (b).

users need to determine the coverage area for each network node if uniform transmission range is not suitable [25]. Our heuristic can provide the lower bound for the transmission range to maintain global connectivity.

5. The minimum energy topology actually forms an energy-efficient backbone. This backbone can be used to facilitate control message dissemination in an efficient way [7].

6. Recently, minimum energy broadcast/multicast is a hot research topic [5], [29]. In these research works, multicasting is realized through pruning a broadcast tree, while a minimum energy broadcast tree is a spanning tree constructed based on the following assumption: Each inner (nonleaf) node transmits with the minimum power to reach all of its children and each leaf node does not transmit and, thus, does not consume energy. According to [23], a sensor consumes almost the same amount of energy when transmitting, idle, and receiving. Thus, actually our strong minimum energy topology is a better model for energy-aware broadcasting and multicasting.

This paper is organized as follows: In Section 2, we first prove that our SMET problem is NP-Complete. Then, we give a very simple incremental power heuristic in Section 3 and compare it with a minimum spanning tree based algorithm by simulation. Finally, in Section 4 , we briefly summarize some related work and conclude our paper in Section 5.

\section{NP-Completeness in Computing a SMET}

A graph is planar if it can be drawn in a plane with no edge crossing. Given an undirected graph $G=(V, E)$, a vertex cover $C$ is a subset of $V$ such that each edge in $E$ is incident to at least one vertex in $C$. A vertex cover problem asks for a vertex cover with minimum cardinality in $G$. Vertex cover, in general graphs is NP-Complete [10]. It is also NPComplete in planar graphs with no vertex degree exceeding four [12]. For convenience, we denote this problem by 4 PlanarVC. We are going to show that a vertex cover in planar graphs with degree at most 3 , denoted by 3 PlanarVC, is also NP-complete.

\section{Lemma 2.1. 3PlanarVC is NP-complete.}

Proof. 3PlanarVC is in NP since its superclass, VC in general graphs, is in NP. Now, we show the Turing reduction from 4PlanarVC to 3PlanarVC.

For a given instance of 4PlanarVC $G=(V, E)$, we construct an instance of 3PlanarVC $G^{\prime}=\left(V^{\prime}, E^{\prime}\right)$ in the following way: Replace each degree 4 vertex $v \in G$ with a widget shown in Fig. 1a. The resultant graph is $G^{\prime}$, with a 


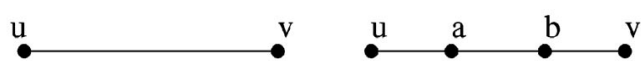

Fig. 2. Each edge $e=(u, v)$ in $G=(V, E)$ is replaced by three edges $(u, a),(a, b),(b, v)$ in $G^{\prime}=\left(V^{\prime}, E^{\prime}\right)$.

degree at most 3 . This construction takes polynomial time.

Note that, for each widget, the minimum number of vertices to cover the six edges formed by $v_{1}, v_{2}, \cdots, v_{7}$, is either $3\left(\left\{v_{2}, v_{4}, v_{6}\right\}\right)$, if none of $\left\{v_{1}, v_{3}, v_{5}, v_{7}\right\}$ is included; or 4 , if at least one of $\left\{v_{1}, v_{3}, v_{5}, v_{7}\right\}$ is included. No vertices other than $v$ in $G$ has influence on the covering of these six edges. In other words, at least four vertices are needed to cover any of the edges in $\left\{\left(b, v_{1}\right),\left(a, v_{3}\right),\left(d, v_{5}\right),\left(c, v_{7}\right)\right\}$ and all of the edges in $\left\{\left(v_{1}, v_{2}\right),\left(v_{2}, v_{3}\right), \cdots,\left(v_{6}, v_{7}\right)\right\}$, and at least three vertices are needed to cover all of the edges in $\left\{\left(v_{1}, v_{2}\right),\left(v_{2}, v_{3}\right), \cdots,\left(v_{6}, v_{7}\right)\right\}$. Thus, we can compute a vertex cover $C^{\prime}$ for $G^{\prime}$ from a vertex cover $C$ of $G$ in the following way: If $v \in C$, put $\left\{v_{1}, v_{3}, v_{5}, v_{7}\right\}$ into $C^{\prime}$, otherwise, put $\left\{v_{2}, v_{4}, v_{6}\right\}$ into $C^{\prime}$. Put all vertices in $C$ with degree $<4$ into $C^{\prime}$. From the above analysis, $C^{\prime}$ is a VC of $G^{\prime}$ if $C$ is a VC of $G$. The size of $C^{\prime}$ is

$$
k-k_{1}+4 k_{1}+3\left(m-k_{1}\right)=k+3 m,
$$

where $k$ is the size of $C, m$ is the number of degree- 4 vertices in $G$, and $k_{1}$ is the number of degree- 4 vertices in $C$.

Conversely, assume we are given a $\mathrm{VC} C^{\prime}$ of $G^{\prime}$ with size $k+3 m$, where $m$ is the number of widget (as shown in Fig. 1) in $G^{\prime}$. Let $C=\phi$. For any widget with vertices $\left\{v_{1}, v_{2}, \cdots, v_{7}\right\}$ in $G^{\prime}$, find the corresponding vertex $v$ in $G$. Note that as elaborated in the previous paragraph, if any of $\left\{v_{1}, v_{3}, v_{5}, v_{7}\right\}$ is in $C^{\prime}$, then four of the seven vertices $\left\{v_{1}, v_{2}, \cdots, v_{7}\right\}$ must be in $C^{\prime}$. Let $k_{1}$ be the number of widgets with this feature. If none of $\left\{v_{1}, v_{3}, v_{5}, v_{7}\right\}$ is in $C^{\prime},\left\{v_{2}, v_{4}, v_{6}\right\}$ must be in $C^{\prime}$. Thus, we can compute $C$ in the following way: For any widget in $G^{\prime}$ with corresponding vertex $v$ in $G$, if at least one of $\left\{v_{1}, v_{3}, v_{5}, v_{7}\right\}$ is in $C^{\prime}$, put $v$ into $C$. Put all vertices in $C^{\prime}$ not belonging to the seven vertices of any widget into $C$. It is clear that $C$ is a VC of $G$ with size $k+3 m-3\left(m-k_{1}\right)-4 k_{1}+k_{1}=k$.

Now, we consider a restricted version of the 3PlanarVC problem: Given a planar graph $G=(V, E)$ satisfying the following two constraints: 1) each node has degree at most 3 and 2) the shortest path (a path with minimum number of edges) between any two degree-3 vertices is at least 3. Compute a vertex cover with minimum size. We denote this problem by $3 r$ Planar $V C$. Note that, in an instance of 3rPlanarVC, the shortest path between two degree- 3 vertices contains at least two degree- 2 vertices.

Lemma 2.2. 3rPlanarVC is NP-Complete.

Proof. 3rPlanar $V C \in N P$ is trivial. Now, we show that 3PlanarVC is polynomial time Turing reducible to 3rPlanarVC.

Consider any instance of 3PlanarVC, denoted by $G=(V, E)$. For each edge $e \in E$, place two Steiner points which divide $e$ into three equal-sized edges, as shown in
Fig. 2. With this modification, we get $G^{\prime}=\left(V^{\prime}, E^{\prime}\right)$, an instance of 3rPlanarVC. This construction takes polynomial time. Now, we show that $G$ has a VC of size $k$ iff $G^{\prime}$ has a VC of size $k+|E|$.

Let $C$ be a VC of $G$ with size $k$. Let $C^{\prime}=\phi$. For each edge $(u, v) \in G$, we have three edges $(u, a),(a, b),(b, v)$ in $G^{\prime}$ as shown in Fig. 2. For each edge $(u, v) \in G$, if $u \in C$, put $b$ to $C^{\prime}$, otherwise, put $a$ to $C^{\prime}$. (Note that we only consider one node $u$ in edge $(u, v)$.) After that, put all vertices in $C$ into $C^{\prime}$. It is clear that $C^{\prime}$ is a $\mathrm{VC}$ of $G^{\prime}$ with size $\left|C^{\prime}\right|=k+|E|$.

Conversely, suppose we are given a $\mathrm{VC} C^{\prime}$ of $G^{\prime}$ with size $k+|E|$. Let $C=\phi$. For every three edges like $(u, a),(a, b),(b, v)$ in Fig. 2, $C^{\prime}$ must contain either $a$ or $b$ or both. If $a \in C^{\prime}$ and $b \notin C^{\prime}$, then $v \in C^{\prime}$ to cover edge $(b, v)$. If $b \in C^{\prime}$ and $a \notin C^{\prime}$, then $u \in C^{\prime}$ to cover edge $(u, a)$. If both $a$ and $b$ are $\in C^{\prime}$, then neither $u$ nor $v$ will be in $C^{\prime}$ since $C^{\prime}$ is minimal. Based on this analysis, we compute $C$ in the following way: let $C=C^{\prime} \cap V$. For every three edges like $(u, a),(a, b),(b, v)$ in Fig. 2, if both $a$ and $b$ are included in $C^{\prime}$, then set $C=C \cup\{u\}$. It is clear that $C$ is a VC of $G$ with size $k$.

\subsection{SMET is NP-Hard}

Now, we are going to show that 3rPlanarVC is polynomial time Turing reducible to our SMET problem. The decision version of 3rPlanarVC is denoted by $\mathcal{P}_{1}$ and is stated below:

$\mathcal{P}_{1}$. Given a planar graph $G=(V, E)$ and a constant $k$, with $G$ satisfying the following two constraints: 1) $G$ has a degree at most 3 and 2) the shortest path between any two degree-3 vertices is at least 3 . Is there a vertex cover of $G$ with a size at most $k$ ?

We use $\mathcal{P}_{2}$ to denote the decision version of our SMET problem:

$\mathcal{P}_{2}$. Given a set of vertices $V^{\prime}$ and a real number $P$, is there a power assignment such that the total energy is at most $P$ ?

We begin with a brief review of several terms in graph theory. Each closed area in a planar graph is called a face. According to Leonhard Euler formula, $f=|E|-|V|+1$, where $f$ is the number of faces in a planar graph. Each face $F$ is associated with $F_{x}$ edges and $F_{x}$ vertices, which form the boundary of the face. We denote the vertex set of $F$ by $\left\{v_{F_{1}}, v_{F_{2}}, \cdots, v_{F_{x}}\right\}$. In an instance of $\mathcal{P}_{1}$, if a face has $y$ neighboring faces, then there are at least $2 y$ degree- 2 vertices on its boundary. Two neighboring faces share at least two degree-2 vertices.

Now, we first construct an instance of $\mathcal{P}_{2}$ (denoted by $V^{\prime \prime}$ ) from any instance of $\mathcal{P}_{1}$ (denoted by graph $G=(V, E)$ ). This construction procedure is relatively complicated. First, we compute a Steiner tree $\tau$ to connect all vertices in $G$. The superposition of $\tau$ and $G$ is a planar graph $G^{\prime}$. Then, we use slightly different strategies to Steinerize edges in $\tau$ and edges in $G$. After this Steinerization, we get an instance of $\mathcal{P}_{2}$ which contains all Steiner points and original vertices in $G$.

\subsubsection{Constructing Steiner Tree $\tau$}

This procedure contains two steps, as shown below:

1. Initally, $\mathcal{F}=\phi$. Start from any face $F$. Add a vertex $g$ in the center of $F$. Draw edges $\left(g, v_{F_{1}}\right),\left(g, v_{F_{2}}\right), \cdots,\left(g, v_{F_{x}}\right)$ 


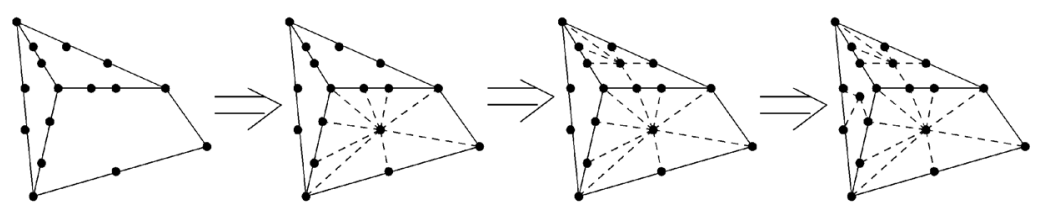

Fig. 3. The step by step demonstration for constructing a Steiner tree to connect all vertices in $G$. In this figure, solid lines represent edges in $G$, while dashed lines represent edges in $\tau$.

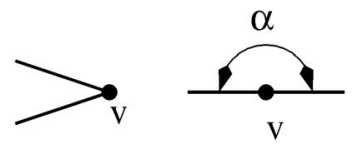

(a)

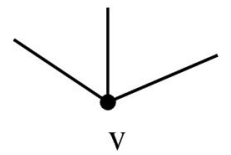

(b)

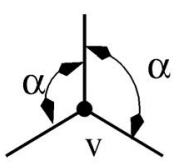

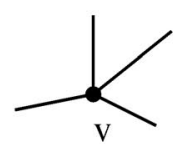

(c)

Fig. 4. Modify nodes with a degree less than 5 in $G^{\prime}$. (a) $v \in G^{\prime}$ has a degree of two, $\alpha=180$ degrees, (b) $v \in G^{\prime}$ has a degree of three, $\alpha=120$ degrees, and (c) $v \in G^{\prime}$ has a degree of four, $\alpha=90$ degrees.

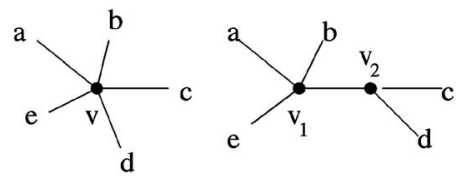

(a)
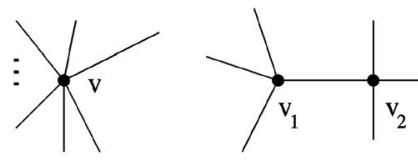

(b)

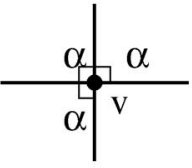

Fig. 5. Widget for transforming a node in $\tau$ with a degree of $\geq 5$. (a) Vertex $v$ has a degree of exactly 5 . (b) Widget when $v$ has a degree of $x \geq 6$. Here, $t=\left\lceil\frac{x-6}{2}\right\rceil+2$.

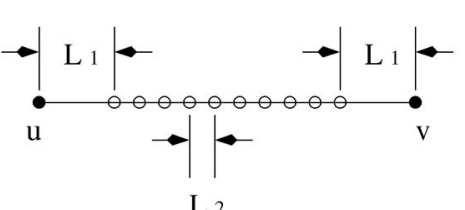

(a)

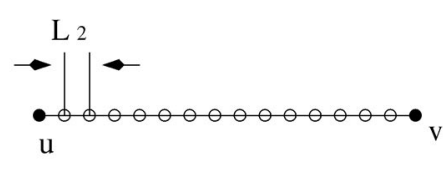

(b)

Fig. 6. (a) $e=(u, v)$ is an edge in the original graph $G$. (b) $e=(u, v)$ is an edge in $\tau$.

to connect $g$ and all vertices associated with $F$. Put $F$ into $\mathcal{F}$.

2. Choose a face $F^{\prime} \notin \mathcal{F}$ satisfying the following constraints: a) $F^{\prime}$ shares a common edge with some face $F^{\prime \prime}$ in $\mathcal{F}$ and b) $F^{\prime}$ has maximum number $(\geq 1)$ of new vertices not on the boundary of any face in $\mathcal{F}$. Add a vertex $g^{\prime}$ inside $F^{\prime}$. Connect $g^{\prime}$ with each of the new vertices on the boundary of $F^{\prime}$. Choose a degree-2 vertex $q$ which is located on the boundary of $F^{\prime}$ and $F^{\prime \prime}$. Note that we can always find this vertex $q$, since $F^{\prime}$ and $F^{\prime \prime}$ share at least three edges as their common boundary. Connect $q$ and $g^{\prime}$. Repeat this step until no $F^{\prime}$ exists.

The Steiner tree $\tau$ connects all vertices in $G$. No edge in $\tau$ overlaps with any edge in $G$. The superposition of $\tau$ and $G$ forms planar graph $G^{\prime}$. The degree of non-Steiner vertices (vertices in $V$ ) in $G^{\prime}$ is at most 4. Each vertex $v \in V$ is incident to exactly one edge in $\tau$, if $v$ has a degree of 3 in $G$; or one or two edges in $\tau$, if $v$ has a degree of 2 in $G$. The example in Fig. 3 demonstrates this construction procedure.

\subsubsection{Steinerizing the Planar Graph $G^{\prime}$}

Before the Steinerization, we need to make some modification to $G^{\prime}$. Note that, with this procedure, bent or curved edges may be introduced. But, the graph remains planar. This modification is stated below:

1. If $v \in G^{\prime}$ has a degree of at most 4, as shown in Fig. 4, we adjust the edges such that angles formed by any two neighboring edges are the same.

2. Other vertices in $G^{\prime}$ must belong to $\tau$ and each has a degree of at least 5 . Replace each of these vertices by a widget as shown in Fig. 5. After this modification, each new vertex has a degree of at most 4 . We now adjust edges such that angles formed by any two neighboring edges are the same, as shown in Fig. 4.

Let $L_{0}^{1}$ be the smallest edge length in the modified $G^{\prime}$. Let $L_{0}^{2}$ be the shortest Euclidean distance between two edges. Set $L_{0}=\min \left\{L_{0}^{1}, L_{0}^{2}\right\}$. Select two numbers $L_{1}$ and $L_{2}$ such that $L_{1}<\frac{1}{3} \cdot L_{0}, L_{2}<L_{1}$. We choose $L_{1}=\frac{1}{4} \cdot L_{0}$. Choose $L_{2}$ such that it is the largest common divisor, which is $<L_{1}$, of all $|(u, v)|-2 \cdot L_{1}$, where $(u, v) \in E$, and all $|(u, v)|$, where $(u, v) \notin E$. Thus, both $|(u, v)|$ and $|(u, v)|-2 \cdot L_{1}$ are multiples of $L_{2}$. (Remember that $E$ is the edge set of the original graph $G$.)

- For each $e=(u, v) \in E$, place equally spaced Steiner points in the middle part of $e$, as shown in Fig. 6a. 

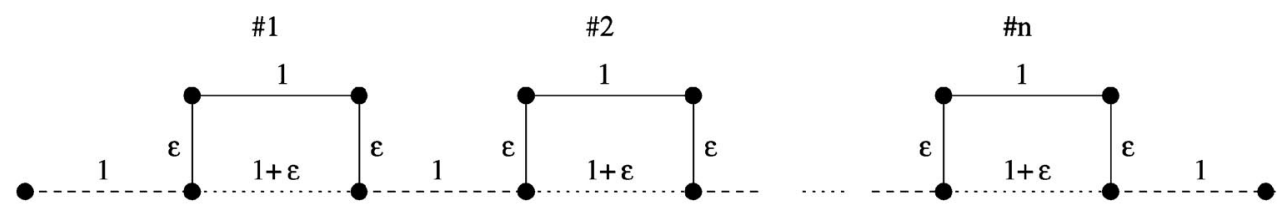

Fig. 7. An example to demonstrate that power assignment based on MST has a performance ratio of exactly 2 .

- $\quad$ For each $e=(u, v) \notin E$, place equally spaced Steiner points on $e$, as shown in Fig. 6b.

We denote this modified and Steinerized graph by $G^{\prime \prime}$. All the vertices in $G^{\prime \prime}$ form an instance of $\mathcal{P}_{2}$, our SMET problem. We denote the vertex set in $G^{\prime \prime}$ by $V^{\prime \prime}$. This construction takes polynomial time.

\subsubsection{Transmit Power Assignment}

Let $C$ be any VC of $G$ with size $k$. For each edge $(u, v) \in E$, choose $u$ if $u \in C$, or $v$ if $v \in C$, but not both, as its cover. In other words, each edge in $G$ has exactly one cover in $C$. Let $p_{v}$ be the power assigned to $v$. Note that, any edge $(u, v) \in E$ in $G$ is divided into many short edges in $G^{\prime \prime}:\left(u, u_{1}\right),\left(u_{1}, u_{2}\right), \cdots,\left(u_{x}, v\right)$, where $u_{x}=\frac{\left(|(u, v)|-2 \cdot L_{1}\right)}{L_{2}}+1$ is the number of Steiner points on $(u, v)$. If $u$ is a cover of $(u, v)$ in $G$, we also say $u$ is a cover of edge $\left(u, u_{1}\right)$ in $G^{\prime \prime}$. Now, we assign energy to each vertex $v^{\prime \prime} \in V^{\prime \prime}$ according to the following rule:

$$
\begin{aligned}
& p_{v^{\prime \prime}}= \\
& \begin{cases}f\left(L_{1}\right) & \left(v^{\prime \prime} \in V \wedge v^{\prime \prime} \in C\right) \vee \\
& \left(v^{\prime \prime} \in V^{\prime \prime}-V, u \in V \wedge u \text { is a cover of edge }\left(u, v^{\prime \prime}\right) \text { in } G^{\prime \prime}\right) \\
f\left(L_{2}\right) & \text { o.w. }\end{cases}
\end{aligned}
$$

It is clear that this assignment generates a strong topology with a total power of

$$
P=(k+|E|) \cdot f\left(L_{1}\right)+\left(\left|V^{\prime \prime}\right|-k-|E|\right) \cdot f\left(L_{2}\right) .
$$

Conversely, if $\forall v^{\prime \prime} \in V^{\prime \prime}$ is assigned a power based on (1), the set $C=\left\{v^{\prime \prime} \mid v^{\prime \prime} \in V \cap V^{\prime \prime} \wedge p_{v^{\prime \prime}}=f\left(L_{1}\right)\right\}$ is a VC of $G$ with size $k$.

\section{Theorem 2.3. SMET is NP-Complete.}

Proof. It is obvious that SMET is in NP. From the above analysis, we know that SMET is NP-hard.

\section{Two Heuristics to Approximate SMET}

SMET is NP-Complete, thus, no efficient exact algorithm exists. The best we can do is to seek fast approximation heuristic with good performance ratio. In this section, we are going to analyze the performance of two heuristics. The first one is based on minimum spanning tree (MST). We assign power to each sensor such that it can reach the farthest neighbor in the tree. The topology generated by this power assignment (only bidirectional links are considered) is globally connected. The second one is an incremental power heuristic. We build a globally connected topology from one node, selecting a node with minimum incremental power to add to the topology at each step. At any time in this heuristic, all bidirectional links in the partial topology form a connected graph. These two heuristics are approx- imation algorithms to SMET. We will compare them through simulation.

\subsection{Minimum Spanning Tree-Based Heuristic}

A naive heuristic is to compute a minimum (edge-cost) spanning tree (MST) and let each sensor transmit at a power that can reach its farthest neighbor in the tree. We call this power assignment based on MST and call this heuristic MST.

Lemma 3.1. Power assignment based on MST has performance ratio 2 .

Proof. Let $P_{M S T}, P_{S M E T}$, and $P_{g S M E T}$ denote the total transmit power assigned based on any minimum spanning tree of the problem instance, in the optimal solution of SMET, and in the optimal solution of gSMET, respectively. Since $P_{M S T} \leq 2 \cdot P_{g S M E T}$ (as proven by [6], [9]) and $P_{g S M E T} \leq P_{S M E T}$, we see $P_{M S T} \leq 2 \cdot P_{g S M E T}$.

We claim that this performance analysis is tight. An example with a tight bound of 2 is shown in Fig. 7. In this example, $\epsilon$ is a sufficiently small positive real number. There are $4 n+2$ nodes in the plane. They form $n$ I-shaped structures connected by edges with a length of 1 . The total power assigned to each node based on the minimum spanning tree (represented by solid and dashed edges) is $4 n \cdot f(1)+2 \cdot f(1)$, while the optimal topology generated by optimal power assignment (represented by dotted and dashed edges, and solid edges with length $\epsilon$ ) has a power of $2 n \cdot f(1+\epsilon)+2 \cdot f(1)+2 n \cdot f(\epsilon)$. When $n \rightarrow \infty, \frac{P_{M S T}}{P_{O P T}}=2$. This indicates that power assignment based on MST has a performance ratio of exactly 2 .

Lemma 3.2. The MST heuristic takes time $O\left(n^{2} \log n\right)$, where $n$ is the number of nodes in the network.

Proof. We need time $O(n)$ to assign transmit power (there are $n$ vertices and $n-1$ edges in the MST, and we need to examine each edge in the tree twice) after the MST is constructed. The famous Kruskal's algorithm and Prim's algorithm both take time $O\left(n^{2} \log n\right)$ to construct a minimum spanning tree. Thus, MST heuristic takes time $O\left(n^{2} \log n\right)+O(n)=O\left(n^{2} \log n\right)$.

\subsection{An Incremental Power Greedy Heuristic}

We propose the following Incremental Power heuristic to approximate the SMET problem. Interestingly, this heuristic gives optimal solution to the example in Fig. 7.

Input: $A$ set $V$ of $n$ sensors in the plane.

Output: A power assignment to each sensor such that the resultant topology is connected and there is at least one bidirectional path between any pair of sensors.

Incremental Power Heuristic: 


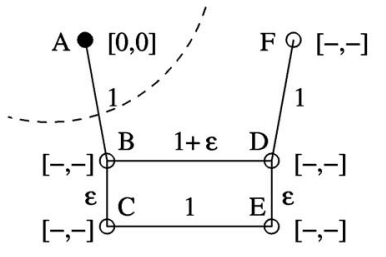

(a)

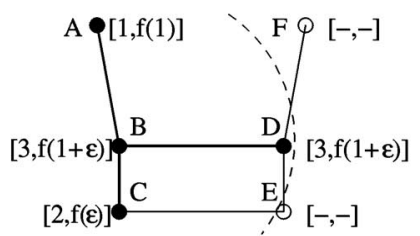

(d)

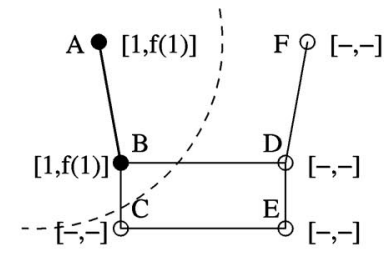

(b)

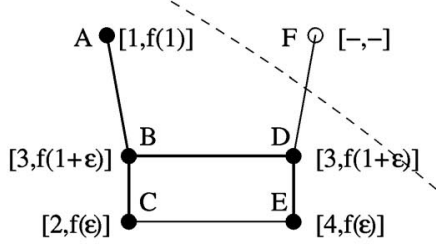

(e)

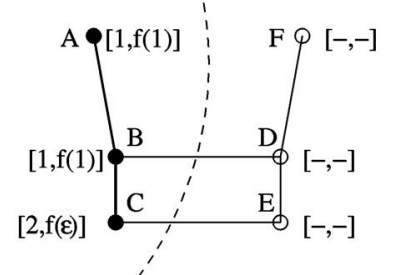

(c)

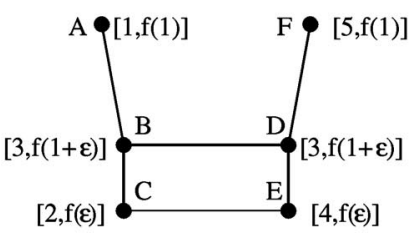

(f)

Fig. 8. An example to demonstrate the incremental power heuristic in a step-by-step way.

1. Initialization. Let $S$ be a set containing the subset of sensors considered so far during the execution of the heuristic. Let $P$ be the total assigned power of all sensors in $S$, and $p_{u}$ be the power expenditure in sensor $u$. Initially, $P=0, S=v_{0}$, where $v_{0}$ is any sensor, $p_{v_{0}}=0$.

2. Let $S^{\prime}=V-S$. Find $u \in S$ and $v \in S^{\prime}$ such that connecting $u$ and $v$ needs minimum incremental power $\delta_{P}$. Here, $\delta_{P}=p_{v}+\delta_{p_{u}}$, where $\delta_{p_{u}}$ is the increased power needed for $u$ to reach $v$ and $p_{v}$ is the power needed to reach $u$ from $v$. Set $S=S \cup\{v\}$, $P=P+\delta_{P}$.

3. If $S=V$, output $P$ and $p_{v}$ for $\forall v \in V$, and then stop; otherwise, go to Step 2.

To demonstrate our algorithm, we provide the following example, as shown in Fig. 8. Note that, in this example, we use vertex label $[x, y]$ at node $v$ to indicate that after step $x$, $v$ 's power is set to be $y$. Edge label $s$ is the Euclidean distance of the edge. $\epsilon$ is a sufficiently small positive real. The corresponding steps are described below:

1. $S=\{A\}, P=0$.

2. $\delta_{P}=2 f(1), S=\{A, B\}$.

3. $\delta_{P}=f(\epsilon), S=\{A, B, C\}$.

4. $\delta_{P}=2 f(1+\epsilon)-f(1), S=\{A, B, C, D\}$.

5. $\delta_{P}=f(\epsilon), S=\{A, B, C, D, E\}$.

6. $\delta_{P}=f(1), S=\{A, B, C, D, E, F\}$.

Note that the total power is $2 f(1+\epsilon)+2 f(1)+2 f(\epsilon)$, while the power assignment based on minimum spanning tree gives $6 f(1)$.

Lemma 3.3. The incremental power heuristic takes time $O\left(n^{3}\right)$, where $n$ is the number of nodes in the network.

Proof. The heuristic takes $n$ steps, thus each edge will be examined at most $n$ times. There are at most $O\left(n^{2}\right)$ number of edges in a network with $n$ nodes, thus the incremental power heuristic takes time $O\left(n^{3}\right)$.

\subsection{Simulation Results}

We compare MST and the incremental power heuristic by simulation. The minimum spanning tree is computed by Kruskal's algorithm. Assume $N$ sensors are randomly distributed in a $1,000 \times 1,000$ square. The power function used in the simulation study is $f(d)=t \cdot d^{\alpha}$, where $\alpha$ is a constant between 2 and 4 . Here, we assume threshold $t$ is the same for every sensor, thus it is set to be 1 in the simulation. For each network size $N$ (in our simulation, $10 \leq N \leq 100$ ), we ran the two heuristics 100 times with different seeds for random number generator. The averaged results for $\alpha=2$ is reported in Fig. 9, which shows that the incremental power heuristic consumes 3.5 percent less energy in average than MST.

Fig. 9 shows that when the network becomes larger, the averaged total energy consumption decreases slowly. This phenomenon is mainly due to two contradicting factors that contribute to energy consumption: network size $N$ and edge length. For denser networks, the edge length is relatively shorter and the averaged energy consumption becomes smaller.

The variance and the maximum of the energy consumption for $\alpha=2$ are plotted in Figs. 10 and 11 to show how stable these two heuristics are. Again, all results are averaged based on 100 runs. In both Figs. 10 and 11, incremental power heuristic is a little bit better than MST. To be specific, the maximum transmit power produced by incremental power heuristic is about 3.3 percent less than that by MST; and the

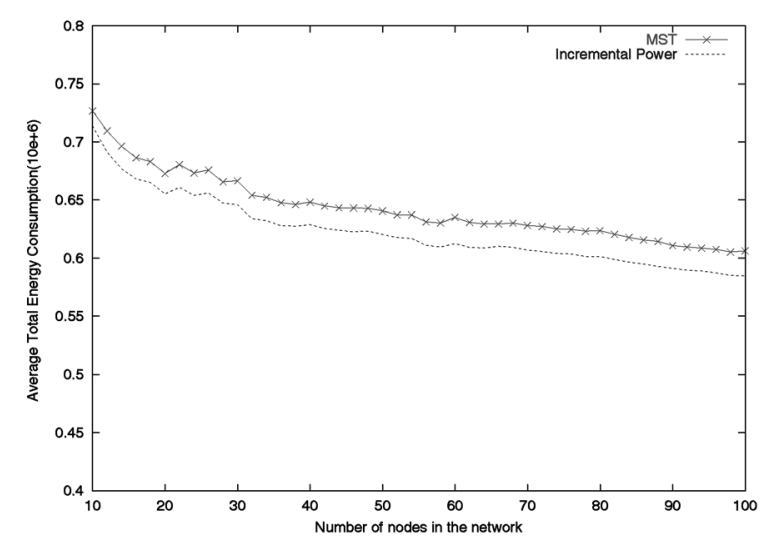

Fig. 9. Simulation results $(\alpha=2)$ on average total energy consumption for MST and the incremental power heuristic. 


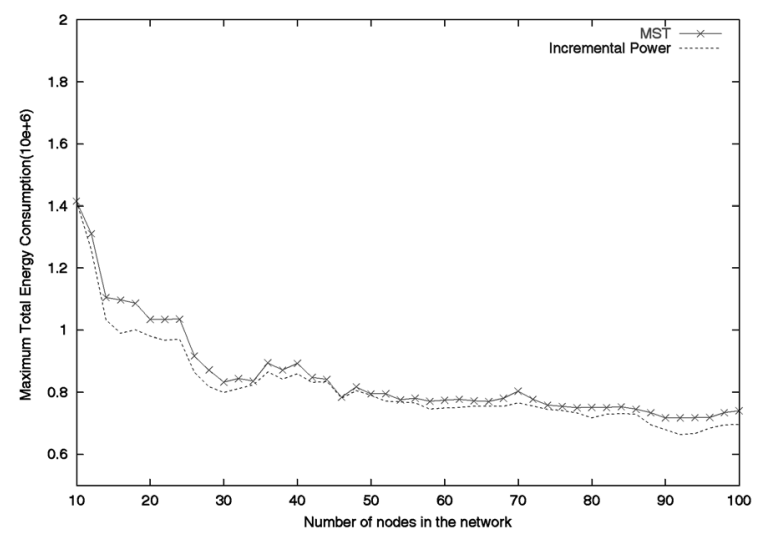

Fig. 10. Simulation results $(\alpha=2)$ on maximum total energy consumption for MST and the incremental power heuristic.

variance from MST is about 8.5 percent more than that from incremental power.

We also notice that both curves for variance and maximum transmit power decrease as the network size increases. This can be explained as follows: If we put $N$ sensors in a $1,000 \times$ 1, 000 square, then when $N$ becomes smaller, the edge length is larger, thus the maximum transmit power is bigger. Also, in sparser networks, the total energy consumption depends more on the deployment of the nodes (the locations or edge lengths of the nodes), thus the variance of the total energy consumption is larger.

For $\alpha=4$, we obtain very similar results for average, maximum, and variance of the total power consumption, except that the two curves tend to be more closer. This is because when $\alpha$ becomes larger, the incremental power heuristic tends to select edges with shorter length, approaching MST.

\section{Related Work}

As we are dealing with the NP-Completeness and heuristics of SMET in this paper, closely related works in literature, to the best of our knowledge, are [6] and [9]. These articles prove (with different Turing reduction scheme) the NPCompleteness of gSMET and show that MST has a performance ratio of 2 for gSMET. However, their proofs are not suitable for SMET, as they are using the "directed graph model" [17], while we need the "undirected graph model" [17].

There exist other works in literature [13], [24], [27], [30] that tackle the topology control problem in wireless sensor networks, but the proposed heuristics have different objectives compared with ours. For example, the heuristics in [24] minimize the maximum transmit power to maintain a globally connected topology; [27] and [30] try to minimize the total power from every sensor to a master site. Algorithms in [24], [27], [30] provide optimal solution with respect to their optimization objectives. The work in [13] tries to balance the node degree in the resultant topology. Our heuristics, MST and incremental power, seek to minimize the total transmit power to maintain a bidirectionally strongly connected topology. These two heuristics can provide approximate solutions to SMET. Now, we

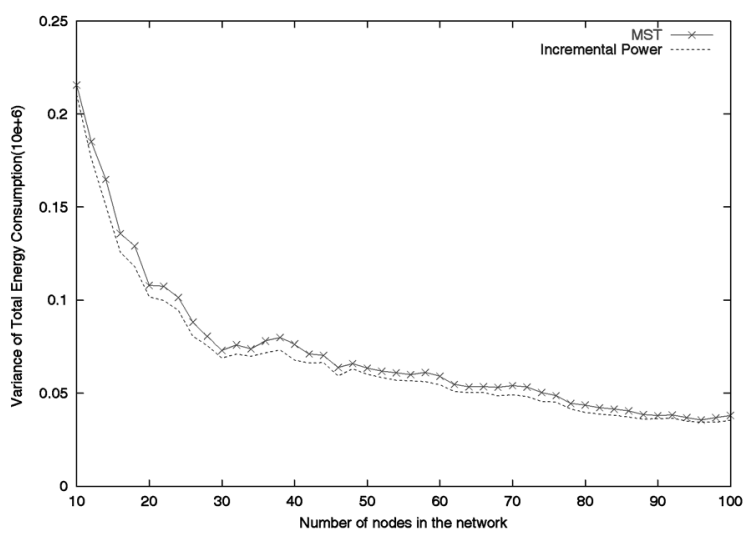

Fig. 11. Simulation results $(\alpha=2)$ on variance of total energy consumption for MST and the incremental power heuristic.

briefly review those related works in the following paragraphs.

Ramanathan and Rosales-Hain [24] show that one can minimize the maximum per node transmit power and maintain global connectivity and biconnectivity. The basic idea is stated below. To guarantee connectivity, a sensor's transmit power only needs to be strong enough to reach all its immediate neighbors in a minimum edge-length spanning tree. With this transmit power, in many cases, the induced graph is a mesh, not a tree, due to the broadcast nature of a wireless sender. Thus, we can adjust each transmit power such that it only reaches a subset of the previous neighbor set, as long as the topology is still connected. This paper [24] also proposes two distributed heuristics for topology control. The first one controls node degree by adjusting transmit power. This heuristic does not guarantee global connectivity. The second one takes advantage of the global information such as node positions collected by network layer routing protocols. This heuristic guarantees a connected network only if the underlying protocol provides up-to-date global information.

The following works try to minimize the total power from every sensor to a master site. Rodoplu and meng [27] assume that each network node knows the position of itself and all its neighbors that can be reached with maximum transmit power. (This neighbor set is denoted by $N$.) Their proposed heuristic is distributed, and contains two phases: First, each node computes the relay set $R$ such that the transmit power is minimized when reaching all nodes in $N-R$ through $R$. This phase determines the transmit power for each node. Next, a directed path with minimum cost (the sum of both transmit and receive powers) to a designated master site from each node is computed by applying the distributed Bellman-Ford algorithm [18]. Wattenhofer et al. [30] proposes a cone-based distributed topology control algorithm, which assumes that nodes can detect each other's direction through message exchange. This algorithm also contains two phases: First, each node increases its transmit power until at least one node is detected in each direction. A direction is defined by a cone with angle $\alpha$. These detected nodes form the neighbor set. In the second phase, each node removes those neighbors from its neighbor set that can be reached by relaying 
through other neighbors. To bound the degree, nodes are removed from the neighbor set even though higher power are needed to reach them by relaying, provided the power increase is not too high. This heuristic guarantees a connected topology if $\alpha \leq \frac{2 \pi}{3}$. Li et al. [16] further analyzes this heuristic and concludes that $\alpha \leq \frac{5 \pi}{6}$ is a necessary and sufficient condition for global connectivity. Borbash and Jennings [4] gives another algorithm for topology control with the help of local and directional information, which is similar to [30]. In the first phase, each node $u$ grows its transmit power until a not-yet-covered node $v_{j}$ is reached. In the second phase, $u$ computes a cone which spans the area covered by $v_{j}$. Repeat these two phases until the $2 \pi$ surrounding area of $u$ is jointly spanned by cones. This algorithm actually computes a Relative Nearest Graph. The topology maintained has good overall performance in terms of interference, reliability, and power usage.

All the above works conduct topology control by adjusting transmit powers. There exist other strategies/ ideas for topology control. For example, $\mathrm{Hu}$ [13] first determines a topology according to Delaunay triangulation. Then, the degree of each node is adjusted through neighbor negotiation such that each node has a similar number of neighbors. This work actually applies heuristics to select logical data links to form a degree-balanced topology. Cheng et al. [8] studies by simulation the effectiveness of decreasing total consumed power to maintain global topology when a very few number of relay sensors are introduced. The objective of their work is to introduce relay sensors to decrease total power. The authors also propose a 2-approximate heuristic to the following network optimization problem: Given a sensor network with fixed transmit power, compute the minimum number of relay sensors needed to maintain the global connectivity.

\section{COnclusion ANd Future Work}

In this paper, we study the SMET problem, which asks for the minimum power assignment to each sensor in a wireless sensor network to maintain global connectivity. SMET considers only bidirectional links, which makes it different from other works in the literature [6], [9], [17]. We show how to formulate our SMET problem and prove its NP-Completeness. We also propose two heuristics, the incremental power and MST, to approximate SMET. Their performances are compared by simulation.

We believe that there are many topology control related works worthy of investigating. It is anticipated that works in [13], [24], [27], [30] may lead to the design of some sound heuristics for SMET. We plan to further explore along this direction and design and evaluate applicable heuristics with better performances for SMET.

We also plan to study various extended SMET problems in the future. We are especially interested in seeking efficient (both distributed and centralized) heuristics for the following problems:

1. Assign minimum power to each sensor in a wireless sensor network such that there are at least $k \geq 2$ disjoint paths between any pair of sensors.
2. Assign minimum power to each sensor in a wireless sensor network such that there are at least $k \geq 1$ disjoint paths between any pair of sensors and the number of neighbors of each sensor is upper bounded by some constant $d_{\max }$.

These problems are harder than SMET. We believe that both of them are significant in helping to design long-lived and survivable sensor networks.

\section{ACKNOWLEDGMENTS}

The authors would like to thank all the anonymous referees and the associate editor for their constructive comments.

\section{REFERENCES}

[1] NS2 page, http://www.isi.edu/nsnam/ns/, 2003.

[2] GloMoSim page, http://pcl.cs.ucla.edu/projects/glomosim/, 2003.

[3] G. Asada et al., "Wireless Integrated Network Sensors: Low Power Systems on a Chip," Proc. European Solid-State Circuits Conf. (ESSCIRC), 1998.

[4] S.A. Borbash and E.H. Jennings, "Distributed Topology Control Algorithm for Multihop Wireless Networks," Proc. 2002 Int'l Joint Conf. Neural Networks, IJCNN, vol. 1, pp. 355-360, 2002.

[5] M. Cagalj, J.-P. Hubaux, and C. Enz, "Minimum-Energy Broadcast in All-Wireless Networks: NP-Completeness and Distributed Issues," Proc. MobiCom 2002, pp. 172-182, 2002.

[6] W.-T. Chen and N.-F. Huang, "The Strongly Connecting Problem on Multihop Packet Radio Networks," IEEE Trans. Comm., vol. 37, no. 3, pp. 293-295, 1989.

[7] X. Cheng, H.D. Du, X. Jia, and G. Xue, "Virtual Backbone-Based Routing in Multihop Ad Hoc Wireless Networks," submitted for publication, 2003.

[8] X. Cheng, D.-Z. Du, L. Wang, and B. Xu, "Relay Sensor Placement in Wireless Sensor Networks," submitted for publication, 2003.

[9] A.E.F. Clementi, P. Penna, and R. Silvestri, "Hardness Results for the Power Range Assignment Problem in Packet Radio Networks," Proc. Third Int'l Workshop Randomization and Approximation in Computer Science (APPROX 1999), pp. 195-208, 1999.

[10] T.H. Cormen, C.E. Leiserson, R.L. Rivest, and C. Stein, Introduction to Algorithms, second ed. MIT Press, 2001.

[11] F.O. Eynde et al., "A Fully-Integrated Single-Chip SOC for Bluetooth," Proc. IEEE International Solid-State Circuits Conf. (ISSCC '01), pp. 196-197, vol. 446, 2001.

[12] M.R. Garey and D.S. Johnson, "The Rectilinear Steiner Tree Problem is NP-Complete," SIAM J. Applied Math., vol. 32, pp. 826834, 1977.

[13] L. Hu, "Topology Control for Multihop Packet Radio Networks," IEEE Trans. Comm., vol 41, no. 10, pp. 1474-1481, 1993.

[14] D.B. Johnson and D.A. Maltz, "Dynamic Source Routing in Ad Hoc Wireless Networks," Mobile Computing, pp. 153-181, T. Imielinski and H. Korth, ed., Kluwer Academic, 1996.

[15] E.S. Jung and N.H. Vaidya, "An Energy Efficient MAC Protocol for Wireless LANs," Proc. INFOCOM '02, vol. 3, pp. 1756-1764, 2002.

[16] L. Li, J.Y. Halpern, P. Bahl, Y.-M. Wang, and R. Wattenhofer, "Analysis of a Cone-Based Distributed Topology Control Algorithm for Wireless Multi-Hop Networks," Proc. 20th ACM Symp. Principles of Distributed Computing (PODC '01), pp. 264-273, 2001.

[17] E.L. Lloyd, R. Liu, M.V. Marathe, R. Ramanathan, and S.S. Ravi, "Algorithmic Aspects of Topology Control Problems for Ad Hoc Networks," Proc. MobiCom '02, pp. 123-134, 2002.

[18] N. A. Lynch, Distributed Algorithms. San Mateo, Cailf.: Morgan Kaufmann, pp. 51-80, 1996.

[19] R. Min, M. Bhardwaj, S.-H. Cho, N. Ickes, E. Shih, A. Sinha, A. Wang, and A. Chandrakasan, "Energy-Centric Enabling Technologies for Wireless Sensor Networks," IEEE Wireless Comm., vol. 9, no. 4, pp. 28-39, 2002.

[20] C.E. Perkins and E.M. Royer, "Ad Hoc On-Demand Distance Vector Routing," Proc. Second IEEE Workshop Mobile Computing Systems and Applications, pp. 90-100, 1999.

[21] G. Pottie and W. Kaiser, "Wireless Sensor Networks," Comm. ACM, vol. 43, no. 5, pp. 51-58, 2000. 
[22] R. Prakash, "Unidirectional Links Prove Costly in Wireless Ad Hoc Networks," Proc. Workshop Discrete Algorithms and Methods for Mobile Computing and Comm. (DIAL-M '99), pp. 15-22, 1999.

[23] V. Raghunathan, C. Schurgers, S. Park, and M. Srivastava, "Energy-Aware Wireless Sensor Networks," IEEE Signal Processing, vol. 19, no. 2, pp. 40-50 2002.

[24] R. Ramanathan and R. Rosales-Hain, "Topology Control of Multihop Wireless Networks Using Transmit Power Adjustment," Proc. INFOCOM, vol. 2, pp. 404-413, 2000.

[25] V. Ramasubramanian, R. Chandra, and R. Mosse, "Providing a Bidirectional Abstraction for Unidirectional Ad Hoc Networks," Proc. INFOCOM '02, vol. 3, pp. 1258-1267, 2002.

[26] T.S. Rappaport, Wireless Communications: Principles and Practice. Prentice Hall, 1996.

[27] V. Rodoplu and T. Meng, "Minimum Energy Mobile Wireless Networks," IEEE J. Selected Areas in Comm., vol. 17, no. 8, pp. 13331344, 1998.

[28] P. Santi, D.M. Blough, and F. Vainstein, "A Probabilistic Analysis for the Range Assignment Problem in Ad Hoc Networks," Proc. MobiHoc '01, pp. 212-220, 2001.

[29] P.-J. Wan, G.C alinescu, X.-Y. Li, and O. Frieder, "MinimumEnergy Broadcast Routing in Static Ad Hoc Wireless Networks," Proc. INFOCOM '01, vol. 2, pp. 1162-1171, 2001.

[30] R. Wattenhofer, L. Li, P. Bahl, and Y.-M. Wang, "Distributed Topology Control for Power Efficient Operation in Multihop Wireless Ad Hoc Networks," Proc. INFOCOM '01, vol. 3, pp. 13881397, 2001.

[31] W. Ye, J. Heidemann, and D. Estrin, "An Energy-Efficient MAC Protocol for Wireless Sensor Networks," Proc. INFOCOM '02, vol. 3, pp. 1567-1576, 2002.

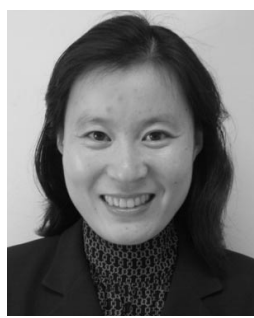

Xiuzhen Cheng received the MS and $\mathrm{PhD}$ degrees in computer science from University of Minnesota at Twin Cities in 2000 and 2002, respectively. She is an assistant professor in the Department of Computer Science at the George Washington University. Her research interests are in mobile ad hoc and sensor networking, and approximation algorithm design and analysis. She is a member of the IEEE.

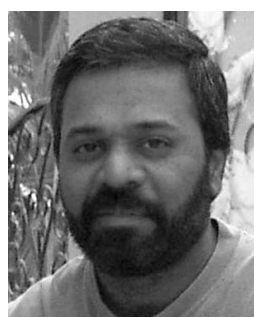

Bhagirath Narahari received the BE degree in electrical engineering from Birla Institute of Technology and Science, Pilani, India, and the MS and PhD degrees in computer and information science from the University of Pennsylvania in 1984 and 1987, respectively. He is a professor in the Department of Computer Science at The George Washington University, where he served as department chair from 1999-2002. His research interests include computer architecture, embedded systems, compiler optimization, power aware computing and communications, distributed systems, and networks.
Rahul Simha received the $\mathrm{PhD}$ degree from the University of Massachusetts, Amherst, in August 1990. He was an assistant then associate professor of computer science at the College of William and Mary from 1990 to 2000. In 2000, he joined the Computer Science Department at The George Washington University as an associate professor. His research interests span a variety of areas including wireless and optical networks, simulation, optimization, and embedded systems. He is a member of the IEEE.

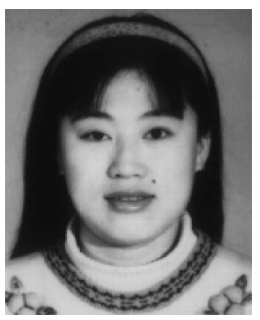

Maggie Xiaoyan Cheng received the BS degree from Beijing University of Aeronautics and Astronautics in 1994. She is currently a PhD student in computer science at the Institute of Technology, University of Minnesota. Her current research interests include mobile computing, ad hoc networking and combinatorial optimization. She is a member of the IEEE.

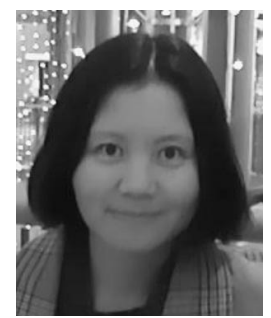

Dan Liu received the MS degree in computer science and engineering from the University of Minnesota in 1998. She is a software engineer at Guidant Co. Currently, she is pursuing the PhD degree in computer science and engineering at the University of Minnesota, Minneapolis. Her research interests are wireless networking and software engineering.

$\triangleright$ For more information on this or any computing topic, please visit our Digital Library at http://computer.org/publications/dlib. 